\title{
Universal Relations in the Attractive Hubbard Model with Next-Nearest Neighbor Hopping
}

\author{
M. BAK \\ Institute of Physics, A. Mickiewicz University \\ Umultowska 85, 61-614 Poznań, Poland
}

Recently a new universal relation between superfluid density $\rho_{\mathrm{s}}$ and $\sigma_{\mathrm{dc}} T_{\mathrm{c}}$, where $\sigma_{\mathrm{dc}}$ is direct current conductivity and $T_{\mathrm{c}}-$ superconducting critical temperature, was found by Homes et al. (Nature 430, 539 (2004)). The theoretical derivation of the relation based on BCS theory is correct only qualitatively. In the present paper this relation is calculated theoretically, using Hartree-Fock approximation and second-order perturbation theory. It is found that although correct qualitatively, quantitatively the results are too small. Inclusion of the second neighbor hopping improves the results.

PACS numbers: 74.25.Fy, 71.10.Fd, 74.20.Fg, 71.27.+a, 72.10.-d

\section{Introduction}

The search for some universal properties of superconducting materials is among many approaches to reveal the mechanisms of high-temperature superconductivity (HTS). One of the earliest universal properties found is so-called Uemura relation [1], a linear scaling between the superfluid density $\rho_{\mathrm{s}}\left(\propto 1 / \lambda^{2}\right.$, where $\lambda$ is a penetration depth) and superconducting transition temperature $T_{\mathrm{c}}-$ valid mostly for underdoped, hole-doped materials. Recently another relation was found by Homes et al. [2] - proportionality between $\rho_{\mathrm{s}}$ and the product $T_{\mathrm{c}} \sigma_{\mathrm{dc}}\left(T_{\mathrm{c}}\right)$, where $\sigma_{\mathrm{dc}}\left(T_{\mathrm{c}}\right)$ is normal state direct current conductivity in temperatures $T \geq T_{\mathrm{c}}$. It is valid among many HTS materials, irrespectively of doping level, doping type (hole-doped or electron-doped), lattice dimensionality; it is also valid for the two BCS superconducting elements $\mathrm{Nb}$ and $\mathrm{Pb}$.

The relation was suggested to be connected with the dirty-limit conductivity [3] but it might also be an effect of several different phenomena instead of the universal one, e.g. Josephson-coupling, marginal Fermi-liquid or $d$-wave pair breaking apart from dirty-limit superconductivity [4].

The theoretical calculations shown in Ref. [3] were based on optical conductivity results in BCS model [5]. The fit of the relation in Ref. [3] was qualitatively correct, although of about twice too large slope in the linear part. The $\mathrm{Nb}$ and $\mathrm{Pb}$ were falling on the theoretical line but the HTS materials were not. 
Reference [6] tries to go one step beyond BCS calculations. While superconducting properties are still calculated in the Hartree approximation to the negative- $U$ Hubbard model, the self-energy in the second-order perturbation theory is included into the calculations of $\sigma_{\mathrm{dc}}$. The limit of infinite dimensions was taken, to suppress vertex corrections and simplify calculations. The resulting curve was qualitatively correct but in this case the slope of the universal part was about twice too small compared to the experimental results (hopping $t^{*}=0.25 \mathrm{eV}$ and $a=4 \AA$ were taken). The present paper extends the analysis of Ref. [6] by considering the effect of next-nearest neighbor hopping.

\section{The method}

The Hamiltonian of the system is negative- $U$ Hubbard model:

$$
H=\sum_{\ll i j \gg \sigma}\left(-t_{i j}-\mu \delta_{i j}\right)\left(c_{i \sigma}^{\dagger} c_{j \sigma}+\text { h.c. }\right)+U \sum_{i} n_{i \uparrow} n_{i \downarrow},
$$

where $\ll i j \gg$ means summation over nearest- (nn) and next-nearest neighbors (nnn), $t_{i j}=t_{1}$ for $\mathrm{nn}$ and $t_{i j}=t_{2}$ for nnn are the hopping integrals, $U=-|U|$ and $\mu$ is a chemical potential. In the reciprocal space the kinetic energy takes the following form:

$$
\epsilon_{k}=-2 t_{1} \sum_{n=1}^{d} \sum_{k} \cos k_{n}-\frac{4 t_{2}}{\sqrt{d-1}} \sum_{n=2}^{d} \sum_{m=1}^{n-1} \cos k_{n} \cos k_{m} .
$$

In $d=\infty$ we must use rescaled hopping $t^{*}=t / \sqrt{2 d}$. The $t^{*}$ unit will be used hereafter. The density of states is given by [7]:

$$
\rho_{0}(\epsilon)=\sqrt{\frac{2}{\pi}} \frac{1}{E} \exp \left(\frac{1-E^{2}}{4 a^{2}}\right) \cosh \frac{E}{2 a^{2}},
$$

where $E(\epsilon)=\sqrt{1+2 a^{2}-2 \sqrt{2} a e}$ and $a \equiv t_{2} / t_{1}$. In infinite dimensions the Green functions and the self-energy depend on the wave vector only by $\epsilon_{k}$, so we use Brullouin-zone-averaged Green functions $G_{\text {loc }}(\omega)=\left\langle G_{k}(\omega)\right\rangle_{k}$ [8]. Density of the single particle excitations is given by:

$$
\rho(\omega)=-\frac{1}{\pi} \operatorname{Im} G_{\mathrm{loc}}\left(\omega+\mathrm{i} 0^{+}\right)=\int_{-\infty}^{\infty} \mathrm{d} \epsilon \rho_{0}(\epsilon) A(\epsilon, \omega),
$$

where $A\left(\epsilon, \omega^{\prime}\right)$ is a standard spectral function of the interacting Green function:

$$
A(\epsilon, \omega)=-\frac{1}{\pi} \operatorname{Im} \frac{1}{\omega-\epsilon+\bar{\mu}-\Sigma\left(\omega+\mathrm{i} 0^{+}\right)} .
$$

The first order self-energy $\Sigma_{1}=U n / 2$ is renormalizing the chemical potential $\bar{\mu}=\mu-\Sigma_{1}$. The $\Sigma$ in Eq. (5) is a second-order self-energy correction, given by a single electron bubble:

$$
\begin{gathered}
\Sigma\left(\mathrm{i} \omega_{n}\right)=-U^{2} \iiint \mathrm{d} \xi_{1} \mathrm{~d} \xi_{2} \mathrm{~d} \xi_{3} \rho\left(\xi_{1}\right) \rho\left(\xi_{2}\right) \rho\left(\xi_{3}\right) \\
\times \frac{f_{1} f_{2}\left(1-f_{3}\right)+\left(1-f_{1}\right)\left(1-f_{2}\right) f_{3}}{\mathrm{i} \omega_{n}-\xi_{1}-\xi_{2}+\xi_{3}},
\end{gathered}
$$


where $f_{n} \equiv f\left(\xi_{n}\right)$ 's are Fermi occupation factors and $\rho(\xi)$ is given by Eq. (4). We iterate Eqs. (4), (5), and (6) until convergence, updating chemical potential $\bar{\mu} \rightarrow \bar{\mu}+\Sigma(\omega=0)$ at every step of iteration to fulfill Luttinger theorem [7]. The calculations were done keeping $\bar{\mu}=0$, which corresponds to the half-filled band for nn hopping $(a=0)$ and to $n$ close to the half-filled band for $a \neq 0$.

The conductivity is given by current-current correlation function, which in $d=\infty$ takes the simplified form [9]:

$$
\sigma(\omega, T)=\int \mathrm{d} \omega^{\prime} \int \mathrm{d} \epsilon \rho_{0}(\epsilon) A\left(\epsilon, \omega^{\prime}\right) A\left(\epsilon, \omega^{\prime}+\omega\right) \frac{f\left(\omega^{\prime}\right)-f\left(\omega^{\prime}+\omega\right)}{\omega} .
$$

Direct current conductivity is given by the $\omega \rightarrow 0$ limit of Eq. (7).

The properties of the superconducting state are calculated in the HartreeFock approximation, by solving the following equations:

$$
\begin{aligned}
& -\frac{1}{U}=\int \rho_{0}(\epsilon) \frac{1}{2 E} \tanh (\beta E / 2) \mathrm{d} \epsilon, \\
& n-1=-2 \int \rho_{0}(\epsilon) \frac{\epsilon-\bar{\mu}}{2 E} \tanh (\beta E / 2) \mathrm{d} \epsilon .
\end{aligned}
$$

where $\beta=1 / k_{\mathrm{B}} T, E=\sqrt{(\epsilon-\bar{\mu})^{2}+\Delta^{2}}$. Limit $\Delta \rightarrow 0$ yields the critical temperature $T_{\mathrm{c}}$.

Superfluid density is given by $\rho_{\mathrm{s}}=-K^{\text {dia }} / 4 \pi^{2}$, where $K^{\text {dia }}$ is small $\boldsymbol{q}$ limit of the Fourier transform of the diamagnetic part of the kernel of linear current response to the external, transverse electromagnetic field; in the ground state:

$$
-K^{\mathrm{dia}}=\int \rho_{0}(\epsilon) \frac{\epsilon(\epsilon-\bar{\mu})}{E} \mathrm{~d} \epsilon .
$$

\section{Results}

In order to compare the results with Ref. [2], the quantities on both axes in Fig. 1 are given in $\mathrm{cm}^{-2}$. Average values of parameters met in HTS materials were used: lattice constant $-4 \AA, t_{1}^{*}=0.25 \mathrm{eV}$ and $a=t_{2}^{*} / t_{1}^{*}=-0.45$ (like in YBCO). Let us note that by definition both $\rho_{\mathrm{s}}$ and $\sigma$ are proportional to the kinetic energy per bond, so they vanish in the $d \rightarrow 0$ limit; thus in fact we calculate the first correction in $1 / d$ expansion: $\mathrm{d} \rho$ and $\mathrm{d} \sigma$.

We can see that performing the calculations in the way described in the present paper and in Ref. [6] underestimates the proportionality constant between $\rho_{\mathrm{s}}$ and $\sigma_{\mathrm{dc}} T_{\mathrm{c}}$. It is to be compared with the results of pure BCS approach (shown in the inset), which overestimate it. Including nnn hopping of the opposite sign to $t_{1}$ improves the situation but only slightly. The largest increase in $\rho_{\mathrm{s}}$ in respect to the case of nn hopping only appears at a nonuniversal part of the curve. The most interesting universal part is connected with large $U$, where the corrections coming from $t_{2}$ diminish. Including 2 nd order perturbation theory to calculate the properties of superconducting state as well as using $T_{\mathrm{c}}$ from Monte Carlo calculations did not change the situation qualitatively [6]. Further calculations, 


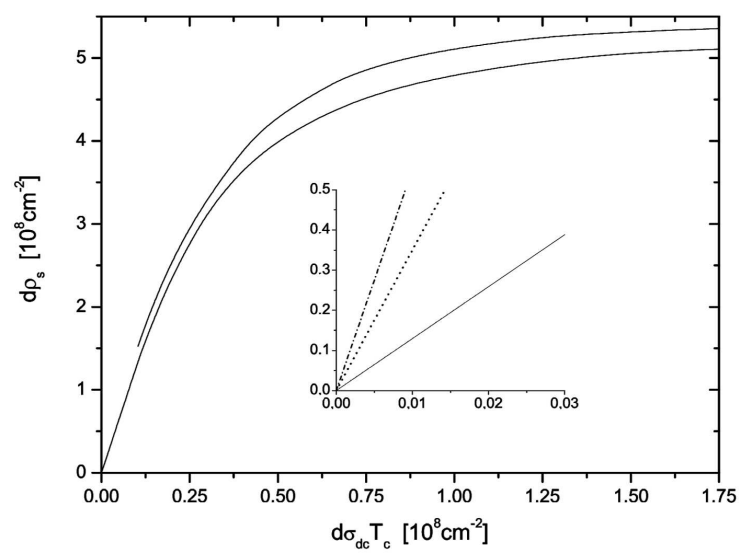

Fig. 1. Superfluid density $\mathrm{d} \rho_{\mathrm{s}}$ vs. $\mathrm{d} \sigma_{\mathrm{dc}} T_{\mathrm{c}}$ for nn hopping only ( $a=0$, lower curve [6]) and for $a=-0.45$ (upper curve). In the inset the enlargement of the area near the axes origin with two curves added: $\mathrm{d} \rho_{\mathrm{s}}=35 \mathrm{~d} \sigma_{\mathrm{dc}} T_{\mathrm{c}}$ (dotted line - HTS materials [2]) and $\mathrm{d} \rho_{\mathrm{s}}=65 \mathrm{~d} \sigma_{\mathrm{dc}} T_{\mathrm{c}}$ (dot-dashed line - BCS theory [2]).

treating both the normal and superconducting states on equal footing are needed. Dynamical mean field theory (DMFT) calculations are in preparation.

\section{Acknowledgments}

Support from the Foundation for Polish Science (FNP) is acknowledged. This work was also supported by the State Committee for Scientific Research (Poland), Project No. 1 P03B 08426.

\section{References}

[1] Y.J. Uemura, G.M. Luke, B.J. Sternlieb, J.H. Brewer, J.F. Carolan, W.N. Hardy, R. Kadono, J.R. Kempton, R.F. Kiefl, S.R. Kreitzman, P. Mulhern, T.M. Riseman, D.Li. Williams, B.X. Yang, S. Uchida, H. Takagi, J. Gopalakrishnan, A.W. Sleight, M.A. Subramanian, C.L. Chien, M.Z. Cieplak, Gang Xiao, V.Y. Lee, B.W. Statt, C.E. Stronach, W.J. Kossler, X.H. Yu, Phys. Rev. Lett. 62, 2317 (1989).

[2] C.C. Homes, S.V. Dordevic, M. Strongin, D.A. Bonn, Ruixing Liang, W.N. Hardy, Seiki Komiya, Koichi Ando, G. Yu, N. Kaneko, X. Zhao, M. Greven, D.N. Basov, T. Timusk, Nature 430, 539 (2004).

[3] C.C. Homes, S.V. Dordevic, T. Valla, M. Strongin, cond-mat/0410719.

[4] J.L. Tallon, J.R. Cooper, S.H. Naqib, J.W. Loram, cond-mat/0410568.

[5] W. Zimmermann, E.H. Brandt, M. Bauer, E. Seider, L. Genzel, Physica C 183, 99 (1991).

[6] M. Bak, submitted to Physica Status Solidi.

[7] E. Muller-Hartmann, Z. Phys. B, Condens. Matter 74, 507 (1989).

[8] E. Muller-Hartmann, Z. Phys. B, Condens. Matter 76, 211 (1989).

[9] T. Pruschke, D.L. Cox, M. Jarrell, Phys. Rev. B 47, 3553 (1993). 